\section{ATH-02 EXTERNAL VALIDATION AND COMPARISON OF OUTCOMES FOR TWO SCORING SYSTEMS FOR LOWER GASTROINTESTINAL BLEEDING}

${ }^{1}$ Christopher Smith, ${ }^{1}$ Gillian Leggett, ${ }^{2}$ Anthoor Jayaprakash, ${ }^{2}$ Mohammed Khan, ${ }^{1}$ John Thomson, ${ }^{1}$ Balasubramaniam Vijayan, ${ }^{1,3}$ Andrew Fraser, ${ }^{1,4}$ John Leeds*. ${ }^{1}$ Aberdeen Royal Infirmary, Aberdeen, UK; ${ }^{2}$ Northumbria Healthcare, Cramlington, UK; ${ }^{3}$ Queen Elizabeth University Hospital, Glasgow, UK; ${ }^{4}$ Freeman Hospital, Newcastle Upon Tyne, UK

\subsection{6/gutjinl-2019-BSGAbstracts.9}

Introduction Lower gastrointestinal bleeding (LGIB) is a common and heterogeneous condition. We have previously reported on a prognostic scoring system devised from the Aberdeen cohort and recently another scoring system has been published (The Oakland score) but requires validation. ${ }^{1}$ Both scoring systems report the ability to identify low risk of rebleeding and mortality at 30 days. This study aimed to compare the two scores particularly with respect to identifying those who may be eligible for safe discharge from a bleeding point of view.

Methods The Aberdeen bleeding unit database was used $(\mathrm{N}=2719)$ to derive and internally and validate our scoring system using the following variables; inpatient status, age, syncope, underlying malignancy, liver disease, blood pressure, pulse rate and haemoglobin. The score was then externally validated in a different region. The Oakland score was then applied to the derivation cohort and receiver operating characteristic (ROC) curves calculated and compared with the Aberdeen score. To determine each scores ability to identify low risk patients, an Aberdeen score of $<1$ was compared with an Oakland score $<9$ (both determined a priori) to assess rebleeding and mortality rates at 30 days.

Results The derivation cohort of 2385 patients (1140 males) was used to compare the two scores of whom $129(5.6 \%)$ patients died within 30 days, 135 (5.7\%) required surgical intervention and $322(13.5 \%)$ experienced re-bleeding. Comparing the two scoring systems with respect to rebleeding showed an area under the ROC curve of 0.742 (0.709 $0.774)$ for the Aberdeen score and 0.687 (0.668 - 0.705) for the Oakland score. With respect to mortality the area under the ROC curve was $0.802(0.755-0.848)$ for the Aberdeen score and $0.757(0.739-0.774)$ for the Oakland score. An Aberdeen score $<1$ was associated with a significantly lower 30 day rebleeding risk compared to an Oakland score <9 (4/ $379(1.1 \%)$ vs. $15 / 355(4.2 \%), p=0.009)$ but 30 day mortality was similar $(0 / 365(0.0 \%)$ vs. $1 / 355(0.3 \%), p=0.493)$.

Conclusion The use of these scores may predict who can be safely discharged. The Aberdeen score is easier to calculate on admission and appears to be superior to the Oakland score, particularly for predicting rebleeding.. Prospective evaluation of both scores is required.

\section{REFERENCE}

1. Oakland K, Jairath V, Uberoi R, Guy R, Ayaru L, Mortensen N, Murphy MF, Collins GS. Derivation and validation of a novel risk score for safe discharge after acute lower gastrointestinal bleeding: a modelling study. Lancet Gastroenterol Hepatol 2017 Sep;2(9):635-643.

\section{ATH-03 EUS TISSUE SAMPLING WITH FORK-TIP BIOPSY NEEDLE IN THE DIAGNOSIS OF TYPE 1 AUTOIMMUNE PANCREATITIS}

${ }^{1}$ Kofi Oppong ${ }^{*}$, 'Pardeep Maheshwari, ${ }^{2}$ Beate Haugk, 'John Leeds, ${ }^{3}$ Matthew Huggettt, ${ }^{3}$ Bharat Paranandi, ${ }^{2}$ Antony Darne, ${ }^{1}$ Manu Nayar. 'HPB Medicine, Freeman Hospital, Newcastle upon Tyne, UK; ${ }^{2}$ Department of Cellular Pathology, Royal Victoria Infirmary, Newcastle upon Tyne, UK; ${ }^{3}$ Department of Gastroenterology, Leeds Teaching hospitals, Newcastle upon Tyne, UK

\subsection{6/gutjnl-2019-BSGAbstracts. 10}

Introduction Endoscopic ultrasound (EUS) fine needle aspiration (FNA) has poor performance in diagnosing autoimmune pancreatitis (AIP) due to small sample size and lack of preserved tissue architecture. In mass forming disease non-diagnostic EUS-FNA may result in a presumptive diagnosis of malignancy and unnecessary surgery. A core biopsy needle with a novel fork-tip design (SharkCore ${ }^{\mathrm{TM}}$ ) has been introduced with the aim of improving yield and maintaining tissue architecture. Literature on its performance in the diagnosis of AIP is limited to a few case reports.

Aim To assess the diagnostic performance of EUS tissue sampling with a fork-tip needle in the diagnosis of type 1 AIP.

Methods Retrospective review of a prospectively maintained AIP database in a tertiary center to identify patients with a final diagnosis of type 1 AIP who underwent EUS-TS during diagnostic workup. Pathology reports were reviewed and classified as per International Consensus Diagnostic Criteria (ICDC); Level 1 (highly suggestive) requires the presence of 3 or all 4 histological features and level 2 (probable) requires 2 features.

Results Between March 2006 and November 2018, 35 procedures were performed on 28 individuals (29 lesions) with a final diagnosis of Type 1 AIP. Mean age ( \pm SD) 63 ( \pm 11.4$)$, 21 male. 29 procedures were for a mass lesion and or biliary obstruction. 2 patients underwent surgical resection and 2 laparoscopic biopsy. There were 8 procedures with an FNA needle, 7 reverse bevel and 20 fork-tip. There were 6 inadequate samples (3 FNA, 2 reverse bevel and 1 fork-tip). Of the 29 adequate samples, $15(51.7 \%)$ met ICDC criteria for diagnosis of AIP all at level 1. 0/13 FNA or reverse bevel samples were diagnostic compared to $15 / 19$ (79\%) fork-tip samples $(\mathrm{p}=0.0001)$. Obliterative phlebitis was identified in $10 / 19$ (52.6\%), storiform fibrosis in 14/19 (70\%), dense lymphoplasmocytic infiltrate in $14 / 19(70 \%)$ and IgG4 positive plasma cell count $>10$ per high power field in $14 / 19(70 \%)$ of adequate fork-tip samples. Of the 17 individuals who underwent 20 procedures (2 repeat and 1 sampling of 2 separate lesions) with a fork-tip needle, 14 (83.2\%) had a final ICDC level 1 diagnostic fork-tip biopsy.

Conclusion In this the largest study to date of the fork-tip core biopsy needle in the diagnosis of AIP, the needle demonstrated very good overall diagnostic performance. This study supports the preferential use of this needle in suspected type 1 AIP. 